\title{
Militärsanitätswesen.
}

\section{Das Militärsanitätswesen in Italien.}

Von Dr. Giuseppe Grieco, Maggiore Medico, attachiert dem Inspektorat des Militärsanitätswesens in Rom.

Militärärzte. Seit 1873 beșteht ein selbständiges militärärztliches Korps (Corpo sanitario militare) mit wirklichen Graden (früher waren sie nur gleichgestellt), sodaß es dieselben Rechte und Pflichten als die kombattanten Offiziere hat (Disziplinarstrafgewalt, Ehrenbezeugungen, Burschen, Urlaub, Beerdigung mit militärischen Ehren, Ehrengerichte, Pension etc.). Diese Aerzte können Zivilpraxis ausüben; deswegen sind sie außer Dienst befugt das Zivilkleid zu tragen. Die Hauptleute - Aerzte der Regimenter, die Stabsoffiziere und die Generalmajore sind berittene Offiziere; die Subalternärzte der berittenen Truppen werden von ihren Truppenteilen beritten gemacht.

Die Militärärzte (in der Regel Hauptleute) nehmen teil an den Sitzungen der Aushebungsräte (Consigli di le va -, für jede Unterpräfektur), um die ärztliche Untersuchung der Wehrpflichtigen auszuführen; sie beschäftigen sich auch mit der Rekrutierung, Ausmusterung etc. der Zollwächter, Gefängniswärter, Schutzmannschaft.

Wenn die Militärärzte ein für jeden Grad bestimmtes Alter erreicht haben (Subalterne 48, Generale $65 \mathrm{Jahre}$ ), müssen sie mit Pension den aktiven Dienst verlassen.

Die Militaritrzte beginnen ihre Karriere mit dem Grade von Sottotenente Medico (Unterleutnantarzt), und sie werden im Bewerbungswege aus den Sottotenenti Medici di Complemento (Ersatzunterleutnantärzte) gewählt (Altersjahre nicht über 30), d. h. aus jenen wehrpflichtigen Doktoren der Medizin und Chirurgie, die den militärärztlichen Unterricht bei der Militärsanitätsapplikationsschule (Scuola d'applicazione di Sanita Militare) zu Florenz erhalten haben. In dieser im Jahre 1883 gestifteten Schule, deren Direktor ein Colonnello Medico (Oberstarzt) ist und deren Lehrer Militärärzte sind, erhalten die Zöglinge durch sieben bis acht Monate Unterricht in Kriegschirurgie, Militärhygiene, forensischer Militärmedizin, chirurgischen Operationen, Epidemiologie, - eine bestimmte Zahl auch Reitunterricht. Am Ende des Kursus müssen die Zöglinge die Prüfungen bestehen, um die Beförderung zu erlangen. Dann werden sie den Truppenteilen beigegeben, um dem Rest ihrer Dienstpflicht zu genügen. In derselben Schule wird auch jährlich ein Kursus der praktischen Hygiene und Bakteriologie für 20 Capitąni Medici (Hauptleuteärzte) gehalten. Die Beförde, rung (promozione) zu Tenente Medico (Leutnantarzt) geschieht ohne Prüfungen, zu Capitano Medico (Hauptmannarzt) und zu Maggiore Medico (Majorarzt) nach Bestehen der Prüfungen uber topographische Anatomie, Kriegschirurgie, chirurgische Operationen, medizinische und chirurgische Klinik etc. nach Wahl (a scelta) oder nach Dienstalter (ad anzianitá), je nach dem Erfolge dieser Prüfungen. Die Beförderung zu Tenente Colonnello Medico (Oberstleutnantarzt) und zu Colonnello Medi co (Oberstarzt) findét ohne Prüfungen nach Dienstalter statt, die zu Maggior Generale Medico (Generalmajorarzt) - der höchste Grad - nur nach Wahl. 
Der Etat der Militärärzte ist folgender:

\begin{tabular}{|c|c|c|c|c|}
\hline & Zahl & $\begin{array}{c}\text { Jahriliches } \\
\text { Gehalt } \\
\text { Lire }\end{array}$ & $\begin{array}{c}\text { Beson- } \\
\text { dere } \\
\text { Zulage } \\
\text { Lire }\end{array}$ & \\
\hline $\begin{array}{l}\text { Sottonenti Medicl } \ldots \ldots \\
\text { Tenenti Medici. . . . . . } \\
\text { Capitant Medicl } \ldots \ldots \\
\text { Magglori Medici } \\
\text { Tenenti Colonnelti Medici } \\
\text { Colonnell Medicl } \\
\text { Maggior Oenerall Medici. }\end{array}$ & $\begin{array}{c}2861) \\
280 \\
71 \\
28 \\
15 \\
3\end{array}$ & $\begin{array}{l}2000 \\
2400 \\
3400 \\
4400 \\
5200 \\
7000 \\
9000\end{array}$ & $\begin{array}{l}300 \\
300 \\
300 \\
300 \\
300 \\
400 \\
400\end{array}$ & $\begin{array}{l}\text { Das Gehalt erfährt für die } \\
\text { Hauptleute und Subaltern- } \\
\text { oftiziere nach 5jăhriger } \\
\text { Dienstzeit in demselben } \\
\text { Grade eine Erhohung um } \\
10 \% \text { für dle ubrigen off- } \\
\text { ziere eine nach 6jăhriger. }\end{array}$ \\
\hline
\end{tabular}

Einer der Generalmajorärzte wird durch königliches Dekret zum Ispettore Capo di Sanità Militare (Chefinspektor des Militarsanitatswesens) ernannt.

Die Militärapotheker (Farmacsti militari) sind Zivilbeamte, vom Kriegsministerium abhängig; sie sind aber gleichgestellt (assimilati a grado militare). Im Kriege und in großen Manövern müssen sie ihre besondere Uniform tragen.

Sanitätsmannschaft. Die Sanitätsmannschaft (Truppa di Sanita) besteht aus 12 Sanitatskompagnien (Compagnie di Sanità), (1' für jedes Armeekorps); sie wird, wie die übrigen Waffengattungen, aus den Wehrpflichtigen rekrutiert, ihr Etat wird vom Kriegsministerium bestimmt; sie versehen den Dienst in den Militärsanitätsanstalten, und ihre Verteilung darin geschieht durch den Colonnello Medico des betreffenden Armeekorps. Die Mannschaften zerfallen in zwei Kategorien, d. i. infermieri (Krankenwärter) und portaferiti (Krankenträger); jene leisten den Dienst bei den Kranken, diese den übrigen allgemeinen Dienst (Magazine, Küche, Bäder etc.). Bei den Sanitätskompagnien genügen ihrer Dienstpflicht auch die einjahrig-freiwilligen Aerzte und Apotheker.

Dienstbetrieb. Das Kriegsministerium ist die oberste Behörde des Sanitätswesens. Dem Kriegministerium als technisch-beratende Behörde ist der Ispettorato di Sanita Militare (Militärsanitłtsinspektorat) beigegeben. Der Ispettorato (aus den $3 \mathrm{Ge}-$ neralmajorärzten, 1 Oberstarztinspekteur, 1 Oberstarzt als Sekretär, 2 Majorarzten, 2 Hauptleutenärzten, 1 Apotheker, dem Inspektorapotheker, 9 Schreibern bestehend) erledigt die vom Ministerium aufgestellten Fragen und macht ihm aus eigener Initiative die den Dienst betreffenden Vorschläge.

Als Organe des Sanitatsdienstes gelten:

a) Der Armeekorps-Sanitätsdirektor (Direttore di Sanità di Corpo d'Armata - Oberstarzt), der mit 1 Hauptmannarzt als Sekretär die Aufsicht des ganzen Sanitätsdienstes des Armeekorps ausübt; gegenüber den Sanitätsanstalten und Sanitätskompagnien hat er die Autorität eines Infanteriebrigadekommandeurs.

b) Die Direktoren der Militärkrankenhäuser, als beratende Behörden der Divisions(Garnisons)kommandeure.

c) Die Aerzte der Truppenteile.

Sanitutsanstalten. Die Sanitätsanstalten bestehen aus:

27 Hauptlazaretten (Ospedali militari principali), 3 Hilfslazaretten (Ospedali militari succursalı), 32 Garnisonskrankenstuben (Infermerie di Presidio), 10 speziellen Krankenstuben (für Militärkollegien, Militärschulen etc.), 6 Genesungsheimen (Reparti di convalescenza), 4 Thermalbadeanstalten (Stabilimenti balneotermali), 1. Trinkkuranstalt (Stabílimento idropinico), Revierkrankenstuben (Infermerie reggimentali). Jode Division besitzt 1 Hauptlazarett (Neapel und Bologna je 2). In Zıvilkrankenhäusern finden Militärkranke ausnahmsweise Unterkunft.

Der Direktor des Hauptlazaretts ist ein Tenente Colonnello Medico, durch königliches Dekret ernannt, der des Hilfslazaretts ein Maggiore Medico, durch ministerielle Verfügung ernannt, der gleichzeitig die technische, administrative und disziplinarische Leitung übernimmt; er besitzt demnach, sei es bezüglich des Personals, Materials oder Dienstbetriebes, die Autorität eines Regiments(resp. Bataillons-) Kommandeurs. Für den administrativen Teil werden dem Direktor, als Prasidenten, ein Verwaltungsrat (Consiglio d'Amministrazione) und einige Offiziere als Rechnungsfuhrer. (Utficiali contabili) zugeteilt und für die Erledigung der Gescháfte ein Hauptmannsarzt als Sekretär (Ajutante Maggiore in $1^{\circ}$ ), der gleichzeitig der Kommandeur der Sanitätskompagnieabteilung am Orte des Hospitals ist.

Die Kranken werden in 4 Stationen (reparti) geteilt (Medizin Chirurgie, Augenkrankheiten und dermo-venerische Krankheiten) Eine besondere Station nimmt die zur Beobachtung geschickten Wehrpflichtigen und Militärs auf, eine andere die an ansteckenden Krankheiten Leidenden einschließlich der Tuberkulósen. Jede Station kann nicht mehr als 60 Kranke aufnehmen. Wenn die Záhl der Kranken gering ist, können mehrere Stationen unter einem Ordinierenden vereinigt werden: sie erhalt den Namen gemischte Station (reparto misto). Ordinierender Arzt jeder Krankenstation ist

1) Die groste Zahl der Sottotenenti besteht aus Sottotenenti Medici di Com. plemento. welche ihrem Dienst genügen. ein Major- oder Huuptmannsarzt (ausnahmsweise ein Subalternarzt), welchem auch Subalternärzte als Assistenten beigegeben werden; Mrlitïrkrankenwärter und Barmherzige Schwestern (Suore di carità) vervollständigen das Personal. Zum Lazarettdienst werden auch in regelmaßigem Wechsel die Truppenärzte kommandiert durch Verfügung des Sanitätsdirektors des Armeekorps. Die Subalternärzte leisten auch den täglichen Wachtdienst (Servizio di guardia), während dessen sie das Lazarett nicht verlassen dürfen.

Der arztliche Besuch bei jeder Station findet zweimal täglich statt (7-8 Uhr morgens, 3-4 Uhr nachmittags).

Die Garnisonskrankenstuben (Infermerie di Presidio) werden der Regel nach in Oertlichkeiten errichtet, wo nicht Zivilkrankenhäuser vorhanden sind; sie funktionieren als kleine Spitäler.

Die Thermalbadeanstalten sind von Juni bis September geöffnet, die zu Ischia auch im Winter. Die Kur dauert 15 Tage. Dasselbe gilt für die 'I'rinkkuranstalt.

In den Revierkrankenstuben (Infermerie reggimentali, infermerie di corpo) - je eine für Regiment oder detachiertes Bataillon - werden nur leichte Krankheiten behandelt, deren Dauer vermutlich 15 Tage nicht übersteigt. Leitender Arzt ist der Hauptmannsarzt des Regiments (resp. Subalternarzt des Bataillons), welchem für die Aufrechthaltung der Ordnung und die nötigen Schreibarbeiten ein Korporalsanitatsgehilfe (Caporale Ajutante di Sanita) beigegeben ist. Arzneien und Verbandmittel werden von der Milıtärapotheke (ausnahmsweise Zivilapotheke) bezogep. Bei den kleinen chirurgischen Operationen gebrauchen die Truppenarzte ihre vorschrittsmäßigen chirurgischen Taschenibestecke. Die Beköstigung der Kranken ist die ordentliche Portion der Mannschatt; die besonderen Spersen (in Ausnahmefallen) werden von dem Marketender (vivandiere) bezogen.

Pflicht der I'ruppenărzte ist auch, den täglichen Besuch der sich krank meldenden Mannschaften, die Impfung und Wiederimpfung auszutühren, den Unterricht den Regimentskrankenträgern (nur im Kriege und in Manövern tätig) zu erteilen, die unentgeltliche Behandlung der kranken Offiziere des betreffenden Truppenteils, die Truppen bei Uebungen, Schieljen etc. zu begleiten, die gute Insandhaltung der Sanitätsausrüstung (Sanitätstornister, Sanitätskotter, Sanitatstaschen etc.) der Truppen, die Ausmusterung der dienstuntaughch gewordenen Mannschatten vorzuschlagen.

Fortbildung. Um die wissenschaftlıchen Kenntnisse der Militärärzte zu tórdern, wird eine bestimmte Zahl von ihnen durch zwei Jahre zu Universitätskliniken kommandiert. Zu diesem Zwecke werden auch bei den Militärlazaretten (gewöhnlich monatliểh) wissenschattliche Versammlungen abgehalten, welchen sämtliche Sanitätsoftiziere der Garnison belwohnen müssen.

Sanitătsoftiziere des Benrlanbtenstandes. Die Sanitătsoffăziere des Beurlaubtenstandes (Ufticiali medici in congedo) umfassen die folgenden 'Kategorien von Santtätsotfizieren:

I. Ufficiali medici di Complemento (Ersatzsanitätsoffiziere)

a) mit dem Grade von Sottotenente: wehrpflichtige Doktoren der Medizin (ehemalige Zöglinge der Militärsanitatsapplikationsschule; eınjährıg-freıwıllige Aerzte; beurlaubte Otfiziere der übrigen Waftengattungen, welche die Doktorwürde erlangt haben). Sie haben sämtlıch dieselbe Dienstpflicht wie die Mannschatten der betreftenden Altersklasse (zwei bıs drei Jahre bei der F'ahne, fünf bis sechs in. der Keserve, vier in der Mobilmiliz, sieben in der Territorialmiliz) bis zum Ende des 39. Jahres. Die einjährig-freswilligen Aerzte werden nach erledigtem Dienste als Sergeanten beurlaubt, und um die Eirnennung zu Sottotenente Medico zu erlangen, müssen sie eıne militärärztlıche Prüfungr bestehen; sie 'müssen 'danin einen dreimonatlichen Dienst leisten;

b) mit ihrem Grade, die noch wehrpflichtigen Sanitätsoffiziere, welche uhren Abschied genommen haben.

II. Sanitätsoffiziere in Hilfsdienststellung '(Ufficiali medici in posizione di servizio ausiliario). Sie rekrutieren sich aus den Sanitatsotfizieren, die den aktiven Diepat intolge des Gesetzes über die Altersgrenze (Subalterne 50, Generalmajore $65 \mathrm{Jahre}$ ) verlassen haben; sie verbleiben in dieser Stellung, in welcher sie immer zur Verfügung der Regierung stehen, drei bis vier Jahre, dann werden sie in die Reserve übersetzt.

III. Sanitätsoffiziere in Stellung von vorläufiger Entlassung (Ufficiali medici in congedo provvisorio). Zu dieser Kategorie gehören die Sanitătsoffıziere (auch übrige Offiziere) im aktiven Dienst, die von der Beforderung auf immer ausgeschlossen, bedingt untanglich sind und welche noch nicht Anspruch aut Pension haben (Dienstalter wenigstens $25 \mathrm{Jahre}$ ). Die Dienstuntauglichkeit wird durch kommissarische ärztliche Untersuchung (visita medica collegiale) festgestellt.

IV. Sanitätsoffiziere der Reserve (Ufficiali medici di Riserva, höchstes Alter: Subalterne 60 Jahre, Generalmajore 75 Jahre). Hierzu werden von Amts wegen die pensionierten 
Sanitatsoffiziere gezåhlt, welche weder der Hilfsdienststellung, den Ersatzoffizieren, noch der Territorialmiliz angehören; auf ihr Gestreh die Sanitätsoffiziere, die den aktiven Dienst ohne Pension verlassen und keine Dienstpflicht mehr haben, die Ersatzoffiziere (uber 40 Altersjahre) und jene der Territorialmiliz, die die Altersgrenze fiir diese Kategorie erreicht haben (58 Jahre für die Stabsoffiziere, 50 Jahre fur die Subalternen). 